EPiC Series in Engineering
Volume 3, 2018, Pages 1624-1630
HIC 2018. 13th International
Conference on Hydroinformatics

\title{
Parameter Uncertainties Assessment in a Conceptual Rainfall-runoff Model using Bayesian paradigm
}

\author{
Tatiane Souza Rodrigues Pereira ${ }^{1}$ Guilherme da Cruz dos Reis ${ }^{2}$, Klebber \\ Teodomiro Martins Formiga ${ }^{3}$ \\ ${ }^{123}$ Universidade Federal de Goiás, Goiânia, Brasil. \\ tatiane.srp@hotmail.com, guilhermecruzreis@hotmail.com, \\ klebber.formiga@gmail.com
}

\begin{abstract}
In this study, the calibration of the rain-flow conceptual model UFGModel1.1 is carried out, and the uncertainties in the predictions of flow rates associated with the parameter set estimates are evaluated by the Generalized Likelihood Uncertainty Estimation (GLUE) and Differential Evolution Adaptive Metropolis (DREAM). The water catchment area of the Botafogo Stream, located in the city of Goiânia, Brazil, was selected as experimental for the development of the study, in which a more distributed spatial discretisation degree (thirteen planes and six channels) was adopted for this basin. The results showed that the various parameter sets were considered optimal, allowing high modelling efficiency, despite the loss of the quality of the simulations and uncertainty increase when using the GLUE.
\end{abstract}

\section{Introduction}

The prediction of hydrological events is important to develop reliable engineering projects, especially about extreme events. In this sense, even if extreme events are natural climatic and watercourse processes, it has been observed that rapid and disordered urban growth has related these events to tragedies in urban environments.

Thus, many hydrological models have been developed and improved over the last decades with a focus on understanding hydrological-hydrological dynamics and quantifying the reliability of hydrological forecasting and uncertainty estimation, so that better decisions can be made for different environments and their particularities (Di Baldassarre \& Montanari, 2009; Kuczera et al., 2006; Liu et al., 2017; Vrugt et al., 2008) 
Thus, there are specificities that are inherent in the modelling processes, in which in relation to the quantification of the error in the application of hydrological models has often been discussed in the scientific field and, since the uncertainty produced during the modelling cannot be avoided, only reduced, has been therefore more frequently studied (Liu et al., 2017). Thus, knowing about the uncertainties produced during modelling has been of great interest to hydrological modellers, especially those who use statistical methods capable of estimating uncertainty (Smith et al., 2015).

This is because unstructured modelling is facilitated or possible through a Bayesian approach. Bayesian inference has already proved to be an essential tool for estimating parameter and uncertainty in modelling as shown by ( $\mathrm{Li} \& \mathrm{Xu}, 2013)$. Therefore, Bayesian statistics are applied in several fields of study to adjust multimodal and complex problems of calibration and optimisation.

The Generalized Likelihood Uncertainty Estimation (GLUE) has shown considerable results in its applications and is still widely used because of its ease of implementation (Beven \& Benley, 1992) However, there are some problems about its Bayesian informality, as it tends to make the statistical inferences considered weaker to summarise the parameters. The popularity of GLUE is attributed to its conceptual simplicity and relative ease of execution, where it can be assumed that many researchers and practitioners will, at least shortly, prefer to continue using simple methods for estimating uncertainty (Vrugt, 2016).

However, there is currently a modern approach that has been widely accepted, the Differential Evolution Adaptive Metropolis (DREAM) (Vrugt et al., 2008; 2009) model, based on the Metropolis method, an evolution of Shuffled Complex Evolution Metropolis (SCEM-SCEM-UA) (Vrugt et al., 2003). The DREAM algorithm presents reliable results, working through a complex scale of sampling, that is, of high dimensionality, keeping the consistency of the natural system behaviour as realistic as possible, thus inducing the minimisation of epistemic errors (Vrugt, 2016).

Although DREAM seems to be a better option to be applied in solving problems than GLUE, its complexity is even more significant. Thus, it is interesting to apply the GLUE to compare the results found for different problems. To evaluate the reliability of the Bayesian GLUE and DREAM approaches, the objective of this study was to compare the uncertainties obtained and the calibration of parameters with the model UFGModel1.1, proposed by (Pereira, 2015) in an urban river basin.

\section{Material and Methods}

The GLUE and DREAM algorithms were applied to determine the parameters of the UFGModel1.1 model, based on kinematic wave equations for planes and channels, and the SCS method for infiltration in the Botafogo Stream basin in Goiânia-GO, Brazil, which has as characteristic almost urbanized entirely, having only $4 \%$ permeable area (Figure 1).

The basin was spatially discretised in thirteen planes and six channels using GIS software. The physical characteristics of the planes and channels were obtained using orthophotos, a plan-altimetric survey with GPS RTK Trimble R6 and a Digital Elevation Model (MDE), a by-product of the Light Detection and Ranging (LiDAR) data, with a resolution of $1 \mathrm{~m}$.

The flow and precipitation data were obtained with the use of pen and pens, in increments of one minute. Eight events were selected and classified, ranging from Forts to Weak (Table 1), four events being used for calibration and four events for validation (Table 2).

The maximum likelihood functions employed were those of Nash and Sutcliffe (1970) and the sum of the Square of Errors, already included in the base of the algorithm DREAM. The development of the model, the implementation of the algorithms and the simulations were performed in MATLAB environment. The Manning roughness coefficient for the permeable area $(0.01-0.2)$, the Manning roughness coefficient for the channel $(0.01-0.2)$, the Initial loss $(1.0$ - 30) and Curve number $(20$ $100)$. 
Parameter Uncertainties Assessment in a Conceptual Rainfall-Runoff Model Using ... T. Pereira et al.

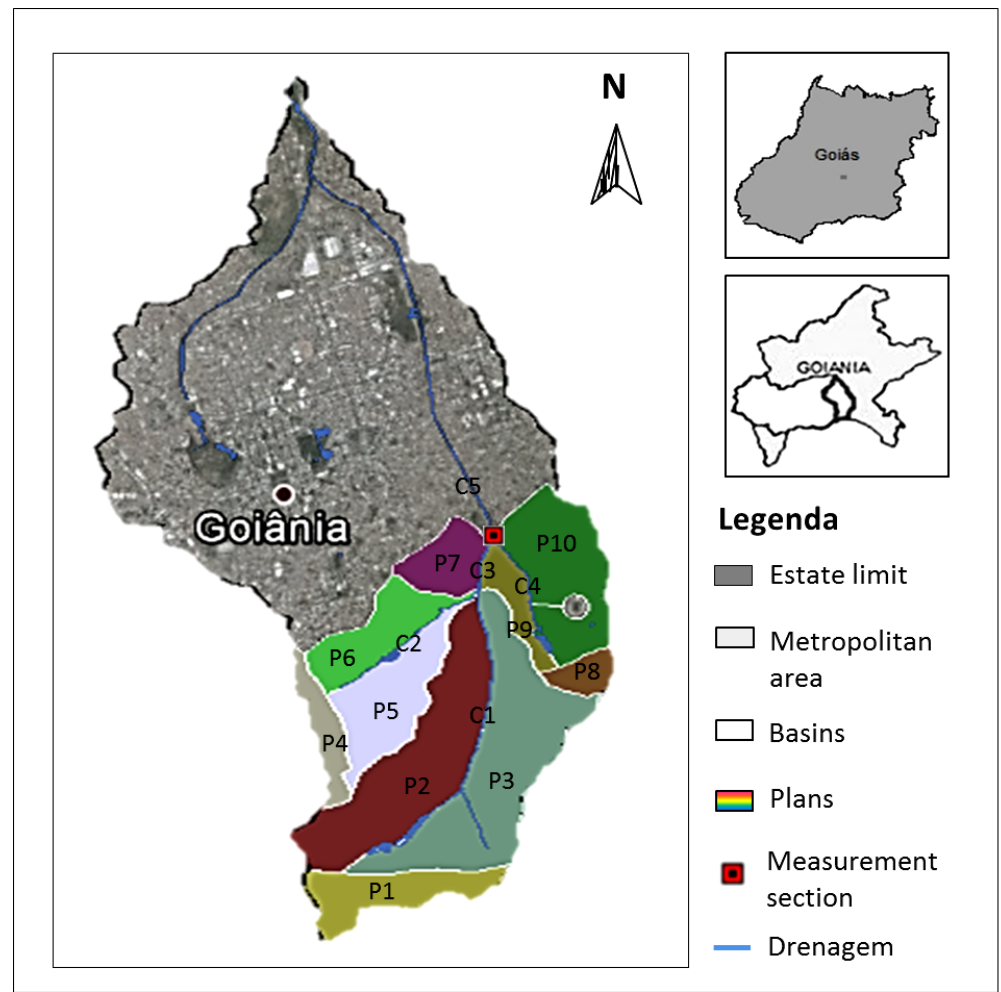

Figura 1: Study area - watershed of the Botafogo Stream.

\begin{tabular}{cc}
\hline Event Classification & Mean Intensity $(\mathrm{mm} / \mathrm{min})$. \\
\hline Forte & $\geq 0,45$ \\
Médio à forte & $0,39-0,44$ \\
Médio & $0,35-0,38$ \\
Médio à fraco & $0,30-0,34$ \\
Fraco & $<0,30$ \\
\hline
\end{tabular}

Table 1: Characterization of selected events.

\begin{tabular}{llccccc}
\hline zEvent/Date & Event Classification & $\begin{array}{c}\text { Int. (max.) } \\
\mathrm{mm} / \mathrm{min} .\end{array}$ & $\begin{array}{c}\text { Int. } \\
(\mathrm{mean}) \\
\mathrm{mm} / \mathrm{min} .\end{array}$ & $\begin{array}{c}\text { Vol. } \\
\text { Total mm }\end{array}$ & $\begin{array}{c}\text { Flow } \\
(\mathrm{max} .) \\
\mathrm{m}^{3} / \mathrm{s}\end{array}$ & $\begin{array}{c}\text { Flow } \\
(\mathrm{méd.)} \\
\mathrm{m}^{3} / \mathrm{s}\end{array}$ \\
\hline $1(12 / 12 / 2013)$ & Strong & 1,80 & 0,57 & 52,98 & 70,59 & 20,76 \\
$2(06 / 03 / 2014)$ & Medium & 0,92 & 0,38 & 27,53 & 31,80 & 13,40 \\
$3(15 / 04 / 2014)$ & Medium to strong & 1,07 & 0,41 & 33,02 & 44,93 & 20,92 \\
$4(06 / 12 / 2013)$ & Weak & 0,78 & 0,27 & 13,34 & 20,56 & 8,73 \\
$5(12 / 11 / 2013)$ & Strong & 1,93 & 0,51 & 50,57 & 65,17 & 23,83 \\
$6(24 / 01 / 2014)$ & Strong & 1,73 & 0,46 & 54,14 & 64,20 & 28,34 \\
$7(24 / 03 / 2014)$ & Medium to strong & 1,26 & 0,42 & 29,49 & 53,61 & 17,84 \\
$8(18 / 12 / 2013)$ & Medium to weak & 1,04 & 0,32 & 16,90 & 31,88 & 12,46 \\
\hline
\end{tabular}

Table 2: Characterization of the events selected for the Botafogo stream in intensities, volumes and flows. 
Parameter Uncertainties Assessment in a Conceptual Rainfall-Runoff Model Using ... T. Pereira et al.

\section{Results and Discussion}

The predictions of the UFGModel1.1 model closely followed the flow observations with both algorithms in the calibration (Figure 1). Qualitatively, the flow uncertainty ranges with the DREAM have always been narrow when compared with those obtained with GLUE. In general, the medians of the predictions of the values of the individual parameters found in the calibration (Table 1), were consistent with the physical characteristics of the experimental basin, where there was a satisfactory adjustment for the different events analysed, and the uncertainties could be obtained numerically.

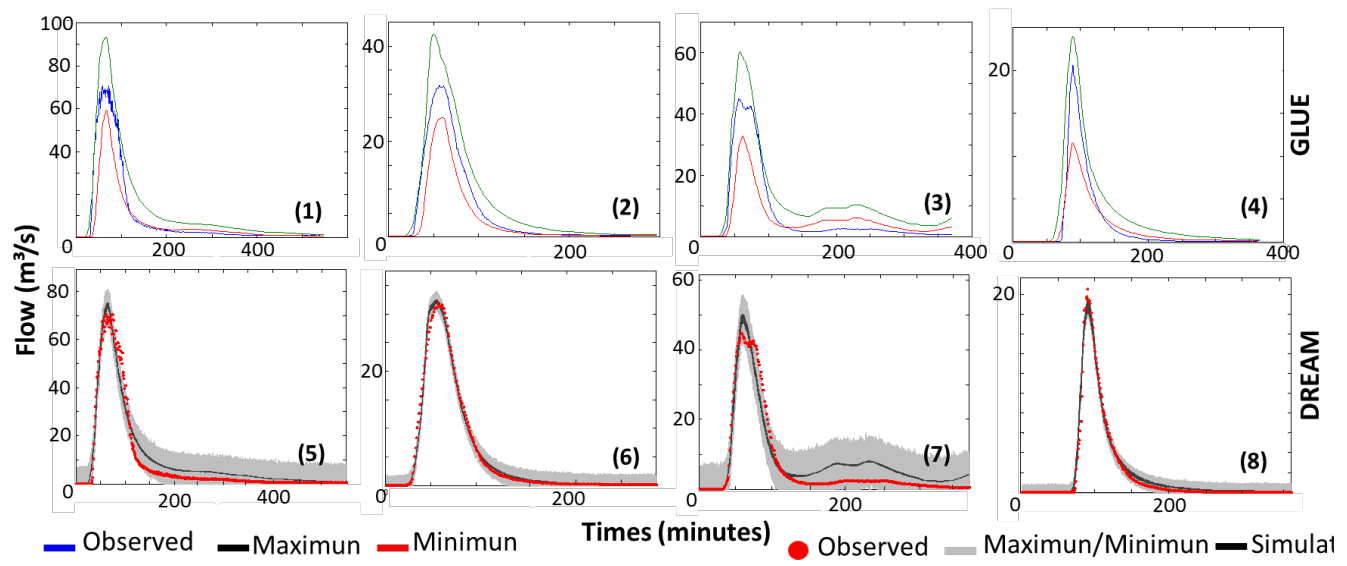

Figura 2: Flow prediction and uncertainty intervals derived with GLUE the DREAM at calibration to the events 1 (Strong), 2 (Medium), 3 (Medium to strong) and 4 (weak), respectively.

The results of the calibration show that the infiltration model met most of the events, with a setting of 5.0 and an average $\mathrm{CN}$ of 86 , values compatible with the soil use conditions of the basin. For events 1 and 4, it was verified that there were events of previous rains, which explains the high values of CN and Manning of the Plan, probably having greater surface flow due to the initial soil moisture condition associated with system deficiency of the region. Channel Manning coefficient was the one that presented the most considerable variation in the results, indicating that the model is less sensitive to this parameter since there is not much change in the likelihood function when its value changes. This can be explained physically because the channel stretch of this basin is relatively small, having little influence on the hydrograph format.

\begin{tabular}{ccccccccc}
\hline \multirow{2}{*}{ Parameters } & \multicolumn{2}{c}{ Event 1 (Strong) } & \multicolumn{2}{c}{ Event 2 (Medium) } & \multicolumn{2}{c}{$\begin{array}{c}\text { Event 3 (Medium to } \\
\text { strong) }\end{array}$} & \multicolumn{2}{c}{ Event 4 (Weak) } \\
\cline { 2 - 9 } & GLUE & DREAM & GLUE & DREAM & GLUE & DREAM & GLUE & DREAM \\
\hline $\begin{array}{c}\text { Manning plan } \\
(\text { s.m-1/3) }\end{array}$ & 0.07 & 0.11 & 0.02 & 0.016 & 0.018 & 0.012 & 0.02 & 0.015 \\
\hline $\begin{array}{c}\text { CN } \\
(\text { dimensionless) }\end{array}$ & 86 & 85 & 87 & 86 & 85 & 79 & 93 & 99 \\
\hline $\begin{array}{c}\text { Ia } \\
\text { (mm/h) }\end{array}$ & 7.0 & 5.4 & 4.0 & 5.1 & 5.0 & 5.0 & 3.0 & 10.5 \\
\hline $\begin{array}{c}\text { Manning channel } \\
\text { (s.m-1/3) }\end{array}$ & 0.05 & 0.005 & 0.07 & 0.12 & 0.05 & 0.197 & 0.08 & 0.197 \\
\hline
\end{tabular}

Table 3: Median of prediction of the values of the individual parameters for the events in calibration.

The validated events (Figure 3) with the following calibration parameter sets with the GLUE and DREAM algorithms, showed that the model works well for simulations with events that use parameters 
Parameter Uncertainties Assessment in a Conceptual Rainfall-Runoff Model Using ... T. Pereira et al.

derived from other events of similar characteristics, even for events classified as weak, except for events with more than one peak. Also in the validation, the associated uncertainties could be quantified numerically for each of the events by the inspection of the hydrograms.

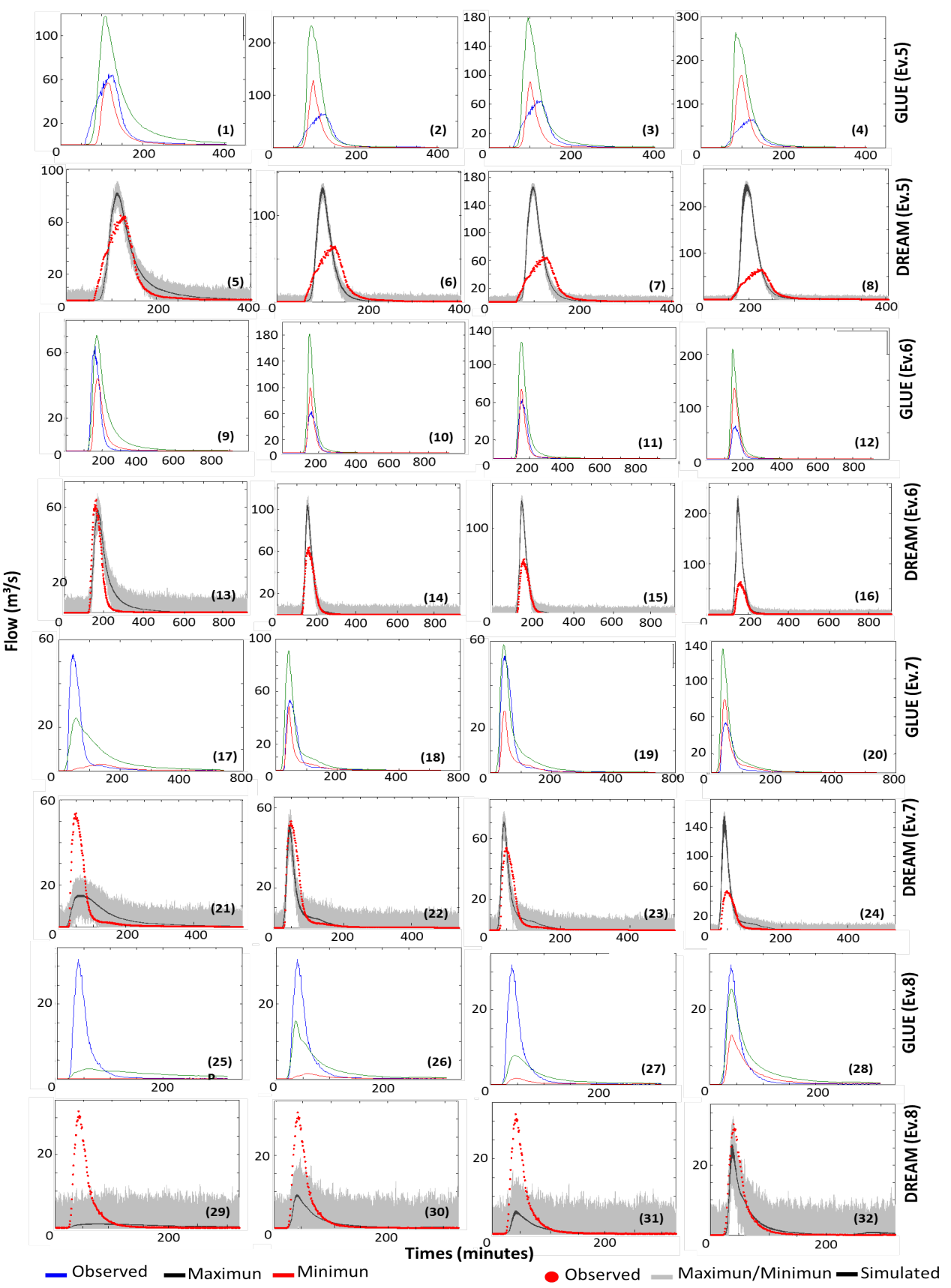

Figure 3: Hydrographs of the simulated flows for the events 5 (weak), 6 (Medium), 7 (weak) and 8 (medium to weak), with each of the parameter sets of events 1,2,3 and 4, respectively, found in calibration with GLUE and DREAM and uncertainty intervals. 
Parameter Uncertainties Assessment in a Conceptual Rainfall-Runoff Model Using ... T. Pereira et al.

Thus, for Event 5, validated with the parameter sets of events 1, 2, 3 and 4, respectively, there are smaller uncertainties with the use of DREAM for validation with the parameter sets of the Event 1 (Figure 3 (5)), where the uncertainty with this algorithm was almost five times lower than with GLUE (Figure 3 (1)), according to the evaluation between the observed and the maximum bands $\left(26.8 \mathrm{~m}^{3}\right.$ However, for the other events, there was no satisfactory validation regarding the adjustment of the peaks of the hydrograms, but in general, there were right adjustments in time. (Fig. 3 (3)). In this case, it is possible to estimate the uncertainty of minimum values of observed values, mainly for the validation with parameter sets of Event 3.

For Event 6, there were similar results to Event 5, in which there was validation with both algorithms with parameter sets of Event 1 (Figure 3 ( 9 and 13)), but with narrower uncertainty ranges with DREAM $\left(15.3 \mathrm{~m}^{3} / \mathrm{s}\right)$ than with GLUE $\left(28.7 \mathrm{~m}^{3} / \mathrm{s}\right)$. For the other events, there was also no satisfactory validation regarding the adjustment of the peaks of the hydrograms. However, there were reasonable adjustments in time. With the GLUE, the minimum bands were also close to the values of observed flows for validation with parameter sets of Event 3 (Figure $3(11)$ ), with a difference of only $11.7 \mathrm{~m}^{3} / \mathrm{s}$ between the simulated and the observed.

For Event 7, there was satisfactory validation with both algorithms using the parameter sets derived from Event 2 (Figure 3 (18 and 22)), where the flows were contained within the narrow uncertainty ranges of the DREAM and presented uncertainty of $44.3 \mathrm{~m}^{3} / \mathrm{s}$ with the GLUE. Also with GLUE, uncertainties were also lower for validation with Event 3 parameter sets than with DREAM (Figure 3 (19 and 23)), with a difference of only $4.9 \mathrm{~m}^{3} / \mathrm{s}$ and $24.4 \mathrm{~m}^{3} / \mathrm{s}$, respectively, between the observed and simulated maximum flow. There was no validation with the set of parameters derived from Event 1 with both algorithms (Figure 3 (17 and 21).) However, there were proper adjustments for the times of most hydrograms. We should point out once again that with GLUE, the minimum bands were also close to the values of observed flows for validation with parameter sets of Event 4 (Figure 3 (20)), with a difference of $25.9 \mathrm{~m}^{3} / \mathrm{s}$ between simulated and observed.

As for Event 8, there was a satisfactory adjustment only for the validation with parameter sets derived from Event 4 (Figure 3 (28 and 32), with lower total uncertainty ranges using DREAM (16.3 $\mathrm{m}^{3} / \mathrm{s}$ ) of (GLUE) and the GLUE $\left(19.5 \mathrm{~m}^{3} / \mathrm{s}\right)$. There was no validation for the simulations with parameter sets derived from Event 1 with the use of both algorithms (Figure 3 (25 and 29)). With the DREAM for the simulations with the parameter sets derived from events 2 and 3 for the hydrograph times, but not for the peaks, because the flow rates were underestimated (Figure $3(26,27,30$ and 31)).

Thus, the results of these analyses using different calibration algorithms and uncertainty analysis show that for the model used, parameter sets derived from strong events validate only strong events, as occurred with events 5 and 6 (Figure 3 (1, 5, 9 and 13)). Meanwhile, the parameter sets derived from medium and medium to strong events tend to validate strong events with GLUE in the minimum range. However, parameter sets derived from weak events do not validate strong events but validate events from medium to strong and medium to weak, and probably weak events.

\section{Conclusions}

The calibrated model acceptably adjusted the observed data, since the efficiency reached was satisfactory in the validation. Although GLUE tends to overestimate flow rates, it is a good analytical tool. However, DREAM is recommended.

The DREAM has been shown to be a better and much more robust method of calibration and assessment of prediction uncertainty than GLUE, but it is also proportionally more complex. In general, DREAM proved to be an excellent calibration tool because it can select sets of solutions with higher quality and with relatively narrow uncertainty limits. The validation was efficient since the model was 
Parameter Uncertainties Assessment in a Conceptual Rainfall-Runoff Model Using ... T. Pereira et al.

able to select expressive sets of satisfactory solutions to represent the observed behaviour with narrow total uncertainty limits, which inspires confidence in the predictions of flow.

The proposed model has shown to be able to provide relatively reliable modelling results when few data are available, which is very common for small urban basins, quantifying numerically expressed uncertainties. However, it is further recommended that a greater number of observed hydrograms be used to verify that they provide better results. This recommendation is made even though the method adopted, considering several sets of parameters to represent the observed behaviour, has proved to be efficient.

It should be noted that this model version uses only four calibration parameters obeying, in addition to the equifinality, the parsimony, so it is quite lean.

\section{References}

Beven, K.J.; Benley, A.M. (1992). The future of distributed models: model calibration and uncertainty prediction. Hydrological Processes, 6(3), (pp. 279-298).

Di Baldassarre, G.; Montanari, A. (2009). Uncertainty in river discharge observations: a quantitative analysis. Hydrology and Earth System Sciences, 13 (pp. 913-921).

Kuczera, G.; Kavetski, D.; Franks, S.; Thyer, M. (2006). Towards a Bayesian total error analysis of conceptual rainfall-runoff models: Characterizing model error using storm-dependent parameters. Journal of Hydrology, 331(1-2) (pp. 161-177).

Li, L.; Xu, C.Y.; Engeland, K. (2013). Development and comparison in uncertainty assessment based Bayesian modularization method in hydrological modeling. Journal of Hydrology, 486 (pp. 384394).

Liu, Y.R.; Li, Y.P.; Huang, G.H.; Zhang, J.L.; Fan, Y.R. (2017). A Bayesian-based multilevel factorial analysis method for analyzing parameter uncertainty of hydrological model. Journal of Hydrology, 553 (pp. 750-762).

Nash, J.E.; Sutcliffe, J.V. (1970). River flow forecasting through conceptual models part I - A discussion of principles. Journal of Hydrology, 10(3), (pp. 282-290).

Pereira, T.S.R. (2015). Modelagem e Monitoramento Hidrológico das Bacias Hidrográficas dos Córregos Botafogo e Cascavel, Goiânia - GO. In: Dissertação de Mestrado do Programa de PósGraduação em Engenharia do Meio Ambiente, Universidade Federal de Goiás - UFG, Goiânia - GO, (pp. 1-133).

Smith, T.; Marshall, L.; Sharma, A. (2015). Modeling residual hydrologic errors with Bayesian inference. Journal of Hydrology, 528 (pp. 29-37).

Vrugt, J.A. (2016). Marcov Monte Carlo Simulation using the DREAM software package: Theory, concepts, and MATLAB implementation. Environmental Modelling \& Software, 75 (pp. 273-316).

Vrugt, J.A.; ter Braak, C.J.; Diks, C.G.; Higdon, D.; Robinson, B.A.; Hyman, J.M.(2009). Accelerating Markov chain Monte Carlo simulation by differential evolution with self-adaptive randomized subspace sampling. International Journal of Nonlinear Sciences and Numerical Simulation, 10(3) (pp. 273-290).

Vrugt, J.A.; ter Braak, C.J.; Clark, M.P.; Hyman, J.M.; Robinson, B.A. (2008). Treatment of input uncertainty in hydrologic modeling: Doing hydrology backward with Markov chain Monte Carlo simulation. Water Resources Research, 44(12) (pp. 1-15 W00B09).

Vrugt, J.A.; Gupta, H.V.; Bouten, W.; Sorooshian, S. (2003). A Shuffled Complex Evolution Metropolis algorithm for optimization and uncertainty assessment of hydrologic model parameters. Journal of Water Resources Research, 39(8) (pp. 1201-1218). 